\title{
THE N2K CONSORTIUM. II. A TRANSITING HOT SATURN AROUND HD 149026 WITH A LARGE DENSE CORE ${ }^{1,2}$
}

\author{
Bun'ei Sato, ${ }^{3,4}$ Debra A. Fischer, ${ }^{5}$ Gregory W. Henry, ${ }^{6}$ Greg Laughlin, ${ }^{7}$ R. Paul Butler, \\ Geoffrey W. Marcy, ${ }^{9}$ Steven S. Vogt, ${ }^{7}$ Peter Bodenheimer, ${ }^{7}$ Shigeru Ida, ${ }^{10}$ Eri Toyota, \\ Aaron Wolf, ${ }^{7}$ Jeff A. Valenti, ${ }^{11}$ Louis J. Boyd, ${ }^{12}$ John A. Johnson, ${ }^{9}$ Jason T. Wright, ${ }^{9}$ \\ Mark Ammons, ${ }^{7}$ Sarah Robinson, ${ }^{7}$ Jay Strader, ${ }^{7}$ Chris McCarthy, ${ }^{5}$ \\ K. L. TAH, ${ }^{5}$ AND Dante Minniti ${ }^{13}$ \\ Received 2005 May 31; accepted 2005 June 28
}

\begin{abstract}
Doppler measurements from Subaru and Keck have revealed radial velocity variations in the $V=8.15$, G0 IV star HD 149026 consistent with a Saturn-mass planet in a 2.8766 day orbit. Photometric observations at Fairborn Observatory have detected three complete transit events with depths of 0.003 mag at the predicted times of conjunction. HD 149026 is now the second-brightest star with a transiting extrasolar planet. The mass of the star, based on interpolation of stellar evolutionary models, is $1.3 \pm 0.1 M_{\odot}$; together with the Doppler amplitude $K_{1}=43.3 \mathrm{~m} \mathrm{~s}^{-1}$, we derive a planet mass $M \sin i=0.36 M_{\mathrm{J}}$ and orbital radius 0.042 AU. HD 149026 is chromospherically inactive and metal-rich with spectroscopically derived $[\mathrm{Fe} / \mathrm{H}]=+0.36, T_{\text {eff }}=6147 \mathrm{~K}, \log g=4.26$, and $v \sin i=6.0 \mathrm{~km} \mathrm{~s}^{-1}$. Based on $T_{\text {eff }}$ and the stellar luminosity of $2.72 L_{\odot}$, we derive a stellar radius of $1.45 R_{\odot}$. Modeling of the three photometric transits provides an orbital inclination of $85^{\circ} .3 \pm 1^{\circ} .0$ and (including the uncertainty in the stellar radius) a planet radius of $(0.725 \pm 0.05) R_{\mathrm{J}}$. Models for this planet mass and radius suggest the presence of a $\sim 67 M_{\oplus}$ core composed of elements heavier than hydrogen and helium. This substantial planet core would be difficult to construct by gravitational instability.
\end{abstract}

Subject headings: planetary systems — stars: individual (HD 149026)

Online material: machine-readable table

\section{INTRODUCTION}

Ongoing precise Doppler surveys by US and European teams are observing about 2000 of the closest and brightest mainsequence stars and have detected more than 150 Jupiter-like extrasolar planets (Marcy et al. 2004; Mayor et al. 2004). These planets show a wide variety in their masses and orbital characteristics. Among them, 25 planets with $M \sin i>0.2 M_{\mathrm{J}}$ reside in orbits close to the central stars with $P<14$ days. Such shortperiod giant planets represented by $51 \mathrm{Peg}$ b (Mayor \& Queloz

\footnotetext{
${ }^{1}$ Based on data collected at the Subaru Telescope, which is operated by the National Astronomical Observatory of Japan.

${ }^{2}$ Based on observations obtained at the W. M. Keck Observatory, which is operated by the University of California and the California Institute of Technology. Keck time has been granted by NOAO and NASA.

3 Graduate School of Science and Technology, Kobe University, 1-1 Rokkodai, Nada, Kobe 657-8501, Japan.

${ }^{4}$ Okayama Astrophysical Observatory, National Astronomical Observatory, Kamogata, Asakuchi, Okayama 719-0232, Japan; satobn@oao.nao.ac.jp.

5 Department of Physics and Astronomy, San Francisco State University, San Francisco, CA 94132; fischer@stars.sfsu.edu.

${ }^{6}$ Center of Excellence in Information Systems, Tennessee State University, 330 10th Avenue North, Nashville, TN 37203; also Senior Research Associate, Department of Physics and Astronomy, Vanderbilt University, Nashville, TN 37235.

${ }^{7}$ UCO/Lick Observatory, University of California, Santa Cruz, CA 95064.

8 Department of Terrestrial Magnetism, Carnegie Institution of Washington, 5241 Broad Branch Road, NW, Washington, DC 20015-1305.

9 Department of Astronomy, University of California, Berkeley, CA 94720.

10 Tokyo Institute of Technology, Ookayama, Meguro-ku, Tokyo 152-8551, Japan; and UCO/Lick Observatory, University of California, Santa Cruz, CA 95064.

${ }^{11}$ Space Telescope Science Institute, 3700 San Martin Drive, Baltimore, MD 21218

${ }^{12}$ Fairborn Observatory, HC2 Box 256, Patagonia, AZ 85624.

13 Department of Astronomy, Pontificia Universidad Católica, Avenida Vicuña Mackenna 4860, Casilla 306, Santiago 200, Chile
}

1995) are called "hot Jupiters." Recently, "hot Neptunes" with $M \sin i=10-20 M_{\oplus}$ and $P<14$ days have also been found (Butler et al. 2004; McArthur et al. 2004; Santos et al. 2004b; Vogt et al. 2005). The very highest Doppler precisions, $\sim 1 \mathrm{~m} \mathrm{~s}^{-1}$, are capable of detecting planets down to about $\sim 5 M_{\oplus}$ in shortperiod orbits (Vogt et al. 2005; Rivera et al. 2005).

Short-period planets have provided deep insights into our understanding of planet formation and the interior structure, atmospheres, and orbital evolution of extrasolar planets. In particular, the detection of a hot Jupiter transiting HD 209458 (Henry et al. 2000b; Charbonneau et al. 2000) has provided key information about the planet radius, density, and atmospheric constituents. The quality of this information is due in large part to the intrinsic brightness of the $\operatorname{star}(V=7.65)$, which permitted photometric precision of $10^{-4}$ using the Hubble Space Telescope (Brown et al. 2001; Wittenmyer et al. 2005).

Atmospheric models predict different radii for planets with and without heavy-element cores. One mystery regarding HD $209458 \mathrm{~b}$ is that the planet radius is $20 \%$ larger than predicted, even by models without cores. This observation has fueled controversy regarding the physical cause of the expansion of the radius of HD 209458b. Burrows et al. (2003) suggest that current theory, including the effects of stellar heating on the atmosphere of the planet but no additional dissipative effects, is consistent with the radius within theoretical and observational error bars. Guillot \& Showman (2002), on the other hand, have calculated that the stellar radiative input of energy generates gas flows from the heated side to the cool side of the synchronously rotating planet. Dissipation of a small fraction of this kinetic energy in the deeper layers of the planet can result in heating and expansion. However, it is not clear why this same mechanism does not inflate the radii of other short-period transiting planets with very similar properties, such as TrES-1 (Alonso et al. 2004). 
Tidal dissipation driven by ongoing eccentricity damping has also been suggested as a source of internal heating in HD 209458b (Bodenheimer et al. 2001, 2003; Baraffe et al. 2003). However, observations of the timing and duration of the secondary eclipse (Deming et al. 2005) and the precise radial velocities (RVs) themselves (Laughlin et al. 2005) constrain the orbital eccentricity to being low, $e<0.02$, making eccentricity damping an unlikely source of significant internal heat for the planet.

One other source for tidal heating has recently been suggested: tidal dissipation driven by gradual coplanarization between the stellar equatorial plane and the orbital plane of the planet. A $4^{\circ}$ misalignment between the stellar equatorial plane and the orbital plane of HD 209458b was determined by modeling the RossiterMcLaughlin (RM) effect (Winn et al. 2005). Since the timescale for coplanarization exceeds the age of the star, this process could still be driving tidal dissipation in the planet. It would be useful to measure this effect in host stars of other transiting planets to determine whether spin-orbit misalignment is uniquely associated with HD 209458.

Because short-period planets can substantially advance our understanding of planet structure and atmospheres, the N2K consortium (Fischer et al. 2005a) was established to carry out a distributed Doppler survey of FGK stars $(7.5<V<10.5)$. As telescope time is allocated, an optimal set of stars is selected and observed over three consecutive nights. Our Monte Carlo tests show that these three observations will flag hot-Jupiter candidates with orbital periods between 1.2 and 14 days. The N2K project draws from a database of $\sim 14,000$ stars closer than $100 \mathrm{pc}$ and brighter than $V=10$. Metallicities for all stars in the database were established using a broadband color calibration (Ammons et al. 2005). Low-resolution spectroscopic follow-up (Robinson et al. 2005) was obtained for stars with high metallicity estimates. High-metallicity stars are preferred for this program because of the well-established planet-metallicity correlation (Gonzalez 1997, 1998, 1999; Gonzalez et al. 2001; Fuhrmann et al. 1997, 1998; Santos et al. 2001, 2003, 2004a; Reid 2002), which shows that stars with $[\mathrm{Fe} / \mathrm{H}]>0.2$ have more than 3 times as many gas giant planets (Fischer \& Valenti 2005). Ida \& Lin (2004) propose that the planet-metallicity correlation is quantitatively accounted for by a core accretion scenario for formation of gas giant planets. Their models also predict the presence of hot Neptunes, albeit only around metal-rich stars.

The N2K consortium has screened $\sim 750$ stars in the past 10 months using the Keck, Magellan, and Subaru telescopes. Three nontransiting close-in gas giant planets have been detected (HD 88133, Fischer et al. 2005a; HD 149143 and HD 109749, Fischer et al. 2005b), and follow-up observations are being made for about 40 additional planet candidates. In this paper, we report on the first detection flagged with Subaru observations: a "hot Saturn" that has been observed to transit the G0 IV star HD 149026.

\section{THE SUBARU N2K SURVEY}

The Subaru component of the N2K survey employs the High Dispersion Spectrograph (HDS) on the $8.2 \mathrm{~m}$ Subaru Telescope (Noguchi et al. 2002). To provide a fiducial wavelength reference for precise RV measurements, we use an iodine absorption cell, which is installed just behind the entrance slit of the spectrograph (Kambe et al. 2002; Sato et al. 2002). We adopt the setup of StdI2b, which simultaneously covers a wavelength region of 3500-6100 $\AA$ by a mosaic of two CCDs and a slit width of 0 ".8, giving a reciprocal resolution $(\lambda / \Delta \lambda)$ of 55,000 . Using this setup, we can obtain a signal-to-noise ratio of $\mathrm{S} / \mathrm{N} \sim 150 \mathrm{pixel}^{-1}$ at $5500 \AA$ for our typical science targets, $V \sim 8.5$ stars, for an exposure time of about $60 \mathrm{~s}$. Such $\mathrm{S} / \mathrm{N}$ and wavelength resolution enable us to achieve a Doppler precision of 4-5 $\mathrm{m} \mathrm{s}^{-1}$.

The set of N2K runs at Subaru were carried out on 2004 July 19-21 and August 23. The first three nights were devoted to identifying stars showing short-period RV variations consistent with a hot Jupiter, and the fourth night was for follow-up observation to eliminate spectroscopic binaries and to extend the observational baseline for previously observed stars. On the first observation, we checked for emission in the $\mathrm{Ca} \mathrm{H}$ and $\mathrm{K}$ lines and analyzed all spectra with a spectral synthesis modeling pipeline to determine metallicity, $T_{\text {eff }}, \log g$, and $v \sin i$ with uncertainties of $0.05 \mathrm{dex}, 40 \mathrm{~K}, 0.05 \mathrm{dex}$, and $0.5 \mathrm{~km} \mathrm{~s}^{-1}$, respectively, as discussed in Valenti \& Fischer (2005). Spectral modeling also flags double-lined spectroscopic binaries (SB2s) by virtue of an extremely poor $\left(\chi_{\nu}^{2}\right)^{1 / 2}$ fit to the observation. Visual inspection of the poorly modeled spectra then confirmed the presence of a second set of spectral lines. As a result of this screening, 9 of 125 stars were identified as SB2s or rapid rotators and were dropped before a second observation was obtained. All information regarding every star observed, including RV measurements, information from spectral synthesis modeling, information about the presence of stellar companions, and chromospheric activity measurements, will appear in an $\mathrm{N} 2 \mathrm{~K}$ catalog (D. A. Fischer et al. 2005, in preparation).

During the Subaru runs described above, we obtained three or four Doppler measurements for 116 stars. Typical instrumental precision of the RVs is found to be $3-4 \mathrm{~m} \mathrm{~s}^{-1}$ with the standard Doppler pipeline developed by Butler et al. (1996). With this Doppler precision, stars displaying rms scatter between 20 and $50 \mathrm{~m} \mathrm{~s}^{-1}$ are likely planet candidates. HD 149026 was one of six stars that showed an interesting initial rms scatter $\left(37 \mathrm{~m} \mathrm{~s}^{-1}\right.$ over three nights in 2004 July). Subsequent observations at Keck in 2005 February and April confirmed a planetary orbit and provided ephemeris times for photometric follow-up.

\section{HD 149026}

HD 149026 is a G0 IV star with $V=8.15$ and $B-V=0.611$. The Hipparcos parallax (Perryman 1997) of 12.68 mas places the star at a distance of 78.9 pc with an absolute visual magnitude $M_{V}=3.66$. Our spectroscopic analysis yields $T_{\text {eff }}=$ $6147 \pm 50 \mathrm{~K},[\mathrm{Fe} / \mathrm{H}]=0.36 \pm 0.05, \log g=4.26 \pm 0.07$, and $v \sin i=6.0 \pm 0.5 \mathrm{~km} \mathrm{~s}^{-1}$. The metallicity prediction from the broadband calibration of Ammons et al. (2005) was $0.36 \pm 0.1$, matching the spectroscopic analysis. From the bolometric luminosity and our spectroscopic $T_{\text {eff }}$, we derive a stellar radius of $1.45 \pm 0.1 R_{\odot}$. The Girardi and Yale evolutionary tracks (DeMarque et al. 2004; Girardi et al. 2000) provided identical stellar mass estimates of $1.3 \pm 0.1 M_{\odot}$. Although the calibration to measure chromospheric activity has not yet been completed for the HDS spectra, no emission was observed in the cores of the $\mathrm{Ca} \mathrm{H}$ and $\mathrm{K}$ lines relative to the NSO solar spectrum (Wallace et al. 1993) shown in Figure 1. The known stellar parameters are summarized in Table 1.

The observation dates, RVs, and instrumental uncertainties for HD 149026 are listed in Table 2. The first four observations were made at Subaru; an offset of $-5.4 \mathrm{~m} \mathrm{~s}^{-1}$ was applied to the Subaru RVs in order to minimize $\left(\chi_{\nu}^{2}\right)^{1 / 2}$ when fitting a Keplerian model to the combined Subaru and Keck velocities. Amazingly, four of seven Keck RVs were serendipitously obtained in transit. Since the transit window is $3 \mathrm{hr}$ long and the orbital period is 2.88 days, there is only a $4.3 \%$ probability that a randomly chosen observation time will occur during transit. The probability of making subsequent in-transit observations is not an 


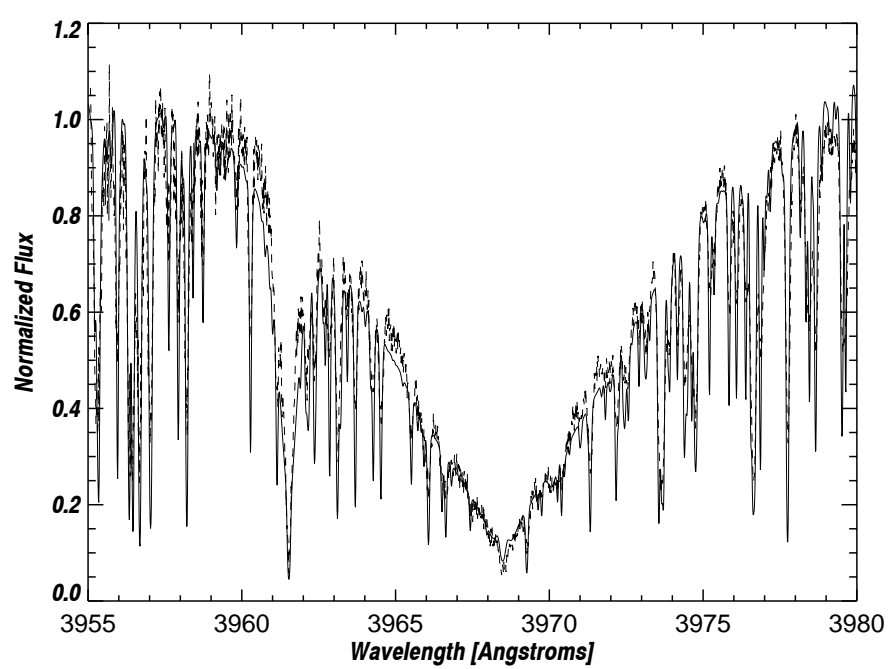

FIG. 1.- Ca H line for HD 149026 plotted as a dashed line, with the NSO solar spectrum overplotted for comparison. No emission is seen in the line core, indicating low chromospheric activity for this star.

independent draw, however, since stars are normally observed near the meridian. Because the orbital period is close to 3 days, observations obtained on three consecutive nights have an enhanced probability of catching two points in transit. Furthermore, two of our in-transit observations were made about an hour apart during a single transit.

Our best-fit orbital parameters are listed in Table 3, and the Keplerian fit is overplotted on the phased RV data in Figure 2. The filled circles represent Subaru RV measurements, and the open diamonds represent Keck data. The four Keck RVs serendipitously taken during transit were removed for purposes of fitting the Keplerian model and are overplotted as triangles in Figure 2. By fitting to the RV data, we obtain a period of $P=$ $2.8766 \pm 0.001$ days. With a fixed circular orbit we measure a velocity amplitude of $43.3 \mathrm{~m} \mathrm{~s}^{-1}$. The rms RV scatter to this fit is $3.8 \mathrm{~m} \mathrm{~s}^{-1}$ and $\left(\chi_{\nu}^{2}\right)^{1 / 2}=1.22$. Uncertainties in the orbital parameters were estimated by running a bootstrap Monte Carlo with 100 trials. With a stellar mass of $1.3 M_{\odot}$, we derive $M \sin i=$ $0.36 M_{\mathrm{J}}$ and $a_{\text {rel }}=0.042 \mathrm{AU}$.

The four RVs of HD 149026 obtained during transit exhibit the RM effect, a deviation from Keplerian velocities that occurs because a transiting planet occults first the approaching limb of the rotating star and then the receding limb of the star. If the orbital plane is coplanar with the stellar equatorial plane, then a

TABLE 1

Stellar Parameters for HD 149026

\begin{tabular}{|c|c|}
\hline Parameter & Value \\
\hline$V \ldots \ldots \ldots$ & 8.15 \\
\hline$M_{V} \ldots \ldots \ldots \ldots$ & 3.66 \\
\hline$B-V \ldots \ldots$ & 0.611 \\
\hline Spectral type ..... & G0 IV \\
\hline Distance $(\mathrm{pc}) \ldots \ldots$ & $78.9 \pm 6.6$ \\
\hline$T_{\text {eff }}(\mathrm{K}) \ldots \ldots \ldots \ldots \ldots$ & $6147 \pm 50$ \\
\hline 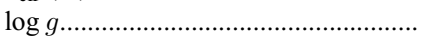 & $4.26 \pm 0.07$ \\
\hline$[\mathrm{Fe} / \mathrm{H}] \ldots \ldots .$. & $0.36 \pm 0.05$ \\
\hline$v \sin i\left(\mathrm{~km} \mathrm{~s}^{-1}\right) \ldots$ & $6.0 \pm 0.5$ \\
\hline 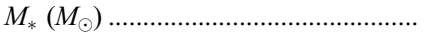 & $1.3 \pm 0.1$ \\
\hline 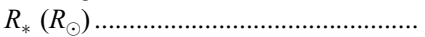 & $1.45 \pm 0.1$ \\
\hline$L_{*}\left(L_{\odot}\right) \ldots \ldots \ldots \ldots \ldots \ldots \ldots$ & $2.72 \pm 0.5$ \\
\hline Age (Gyr) & $2.0 \pm 0.8$ \\
\hline
\end{tabular}

TABLE 2

Radial Velocities for HD 149026

\begin{tabular}{|c|c|c|c|}
\hline $\begin{array}{l}\text { Observation Date } \\
(\mathrm{JD}-2,440,000)\end{array}$ & $\begin{array}{l}\text { Radial Velocity } \\
\quad\left(\mathrm{m} \mathrm{s}^{-1}\right)\end{array}$ & $\begin{array}{l}\text { Uncertainties } \\
\left(\mathrm{m} \mathrm{s}^{-1}\right)\end{array}$ & Observatory \\
\hline $13,206.913796 \ldots \ldots \ldots \ldots$ & -24.10 & 3.15 & Subaru \\
\hline $13,207.935648 \ldots \ldots \ldots \ldots$ & 38.95 & 3.21 & Subaru \\
\hline $13,208.908681 \ldots \ldots \ldots \ldots$ & -42.48 & 3.10 & Subaru \\
\hline $13,241.797685 \ldots \ldots \ldots \ldots$ & 6.06 & 4.61 & Subaru \\
\hline $13,427.158623 \ldots \ldots \ldots \ldots$ & 0.76 & 2.08 & $\mathrm{Keck}^{\mathrm{a}}$ \\
\hline $13,429.113935 \ldots \ldots \ldots \ldots$ & 27.76 & 2.29 & Keck \\
\hline $13,430.079410 \ldots \ldots \ldots \ldots$ & -0.82 & 2.02 & $\operatorname{Keck}^{\mathrm{a}}$ \\
\hline $13,430.095787 \ldots \ldots \ldots \ldots$ & -8.66 & 2.15 & Keck $^{\mathrm{a}}$ \\
\hline $13,479.036481 \ldots \ldots \ldots \ldots$ & -18.49 & 3.36 & $\mathrm{Keck}^{\mathrm{a}}$ \\
\hline $13,480.110602 \ldots \ldots \ldots \ldots$ & -31.13 & 2.41 & Keck \\
\hline $13,483.930012 \ldots \ldots \ldots \ldots$ & 30.55 & 3.27 & Keck \\
\hline
\end{tabular}

${ }^{a}$ RVs serendipitously obtained during transit.

symmetrical deviation occurs about mid-transit. The observation of this effect in the Doppler velocities provides an unambiguous and independent confirmation of the photometric planet transit. The amplitude of this effect in HD 149026 can be estimated by scaling the effect in HD 209458, which we estimate at $\Delta v=45 \mathrm{~m} \mathrm{~s}^{-1}$. The photometric transit depth is only $0.003 \mathrm{mag}$ in HD 149026, compared to 0.019 mag in HD 209458; however, the rotational velocity is somewhat higher (6 compared to $4 \mathrm{~km} \mathrm{~s}^{-1}$ ). So, we expect a maximum RM amplitude of about $(3 / 19)(6 / 4)(45)=11 \mathrm{~m} \mathrm{~s}^{-1}$, consistent with our four in-transit observations. If the radius of HD 149026b were similar to HD 209458b, the departure from a Keplerian model would have precluded a quick detection. Asymmetry in the RM velocities arises from non-coplanar orientations between the stellar equatorial plane and the orbital plane. While the in-transit RVs plotted in Figure 2 seem to show an asymmetry, fits that include the constraints provided by photometry indicate that this asymmetry is only marginally significant.

The stellar radius is a critical parameter since only the ratio of the planet radius to the stellar radius is well determined by the transit depth. We determine the stellar radius from the Hipparcosbased stellar luminosity, $2.72 \pm 0.5 L_{\odot}$, and our spectroscopically derived $T_{\text {eff }}, 6147 \pm 50 \mathrm{~K}$ :

$$
\frac{R}{R_{\odot}}=\sqrt{\frac{L}{L_{\odot}}}\left(\frac{T_{\odot}}{T_{\text {eff }}}\right)^{2}=1.45 \pm 0.1 .
$$

Uncertainty in the stellar radius is dominated by the uncertainty in the stellar luminosity. The limiting factor for precision

TABLE 3

Spectroscopic Orbital Solution for HD 149026b

\begin{tabular}{|c|c|}
\hline Parameter & Value \\
\hline$P($ days $) .. . \ldots \ldots \ldots$ & $2.8766 \pm 0.001$ \\
\hline 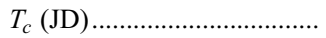 & $2,453,317.838 \pm 0.003$ \\
\hline Eccentricity ........................... & 0 (fixed) \\
\hline$K_{1}\left(\mathrm{~m} \mathrm{~s}^{-1}\right) \ldots \ldots \ldots \ldots \ldots \ldots \ldots \ldots \ldots \ldots \ldots \ldots$ & $43.3 \pm 1.2$ \\
\hline 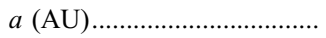 & 0.042 \\
\hline$a_{1} \sin i(\mathrm{AU}) \ldots \ldots \ldots \ldots \ldots \ldots \ldots \ldots \ldots \ldots$ & $1.037 \mathrm{E}-5$ \\
\hline 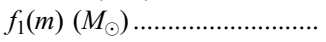 & $1.839 \mathrm{E}-11$ \\
\hline$M \sin i\left(M_{\mathrm{J}}\right) \ldots \ldots \ldots \ldots \ldots \ldots \ldots \ldots \ldots \ldots \ldots$ & $0.36 \pm 0.03$ \\
\hline 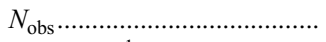 & 7 (out of transit) \\
\hline $\mathrm{rms}\left(\mathrm{m} \mathrm{s}^{-1}\right) \ldots \ldots \ldots \ldots \ldots \ldots \ldots \ldots \ldots \ldots \ldots \ldots$ & 3.8 \\
\hline Reduced $\left(\chi_{\nu}^{2}\right)^{1 / 2}$. & 1.22 \\
\hline
\end{tabular}




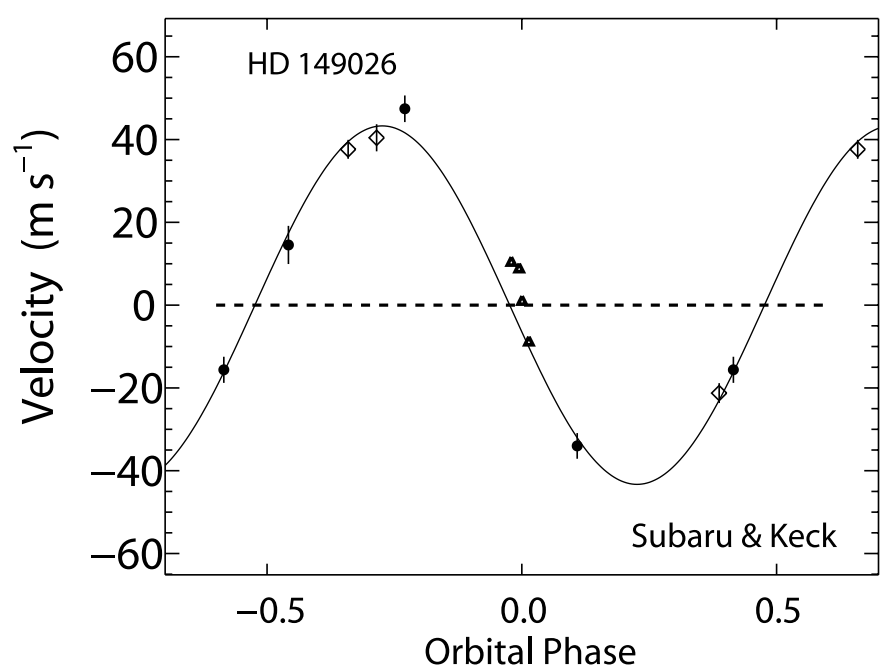

FIg. 2.-Phased RVs for HD 149026 from Subaru ( filled circles) and Keck (open diamonds). The triangles represent RV measurements made at Keck during transit. These velocities exhibit the RM effect and were removed when fitting a Keplerian model. While there appears to be an asymmetry in the intransit velocities, fits that include the constraint provided by the observed transit midpoint indicate that this asymmetry is only marginally statistically significant. With an orbital period of 2.8766 days, velocity amplitude of $43.3 \mathrm{~m} \mathrm{~s}^{-1}$, and stellar mass of $1.3 M_{\odot}$, the implied planet mass is $M \sin i=0.36 M_{\mathrm{J}}$ and the orbital radius is $0.042 \mathrm{AU}$.

of the stellar luminosity is the Hipparcos parallax precision of 1 mas. Higher precision astrometry for HD 149026 could substantially improve the precision in the stellar radius. For example, the Space Interferometry Mission (SIM) should achieve better than $10 \mu$ as single-measurement precision (Shao 2004), significantly reducing the uncertainty in the stellar luminosity and improving the measurements of stellar and transiting planet radii by a factor of 6 .

\section{PHOTOMETRIC RESULTS FOR HD 149026}

We began photometric observations of HD 149026 with the T11 $0.8 \mathrm{~m}$ automatic photometric telescope (APT) at Fairborn Observatory after the star was recognized as a short-period RV variable. Precision photometric measurements are an important complement to Doppler observations and can help to establish whether the RV variations are caused by stellar magnetic activity or planetary-reflex motion (Henry et al. 2000a). For example, Queloz et al. (2001) and Paulson et al. (2004) have shown that photospheric features were the source of periodic RV variations in the young G0 V star HD 166435 and in several Hyades dwarfs. In addition, photometric observations can detect possible transits of the planetary companions and so allow the determination of their radii and true masses (e.g., Henry et al. 2000b).

The T11 APT is equipped with a two-channel precision photometer employing two EMI 9124QB bi-alkali photomultiplier tubes to make simultaneous measurements in the Strömgren $b$ and $y$ passbands. This telescope and its photometer are essentially identical to the T8 $0.8 \mathrm{~m}$ APT and photometer described in Henry (1999). The APT measures the difference in brightness between a program star and a nearby constant comparison star with a typical precision of $0.0015 \mathrm{mag}$ for bright stars $(V<$ 8.0). For HD 149026, we used HD $149504(V=6.59, B-V=$ $0.44, \mathrm{~F} 5)$ as our primary comparison star, which was shown to be constant to $0.002 \mathrm{mag}$ or better by comparison with two additional comparison stars (HD 146135, $V=7.08, B-V=$ 0.36, F2; HD 151087, $V=6.02, B-V=0.32$, F2.5 III-IV). We reduced our Strömgren $b$ and $y$ differential magnitudes
TABLE 4

Photometric Observations of HD 149026

\begin{tabular}{|c|c|}
\hline $\begin{array}{l}\text { Observation Date } \\
(\mathrm{HJD}-2,400,000)\end{array}$ & $\begin{array}{l}\Delta(b+y) / 2 \\
\quad(\mathrm{mag})\end{array}$ \\
\hline $53,485.7969 \ldots \ldots \ldots \ldots \ldots \ldots$ & 1.6056 \\
\hline $53,485.8832 .$. & 1.6031 \\
\hline $53,486.7939 \ldots \ldots \ldots \ldots \ldots \ldots \ldots \ldots \ldots$ & 1.6035 \\
\hline $53,486.8799 \ldots \ldots \ldots \ldots \ldots \ldots \ldots \ldots$ & 1.6036 \\
\hline $53,487.7216 \ldots \ldots \ldots \ldots \ldots \ldots \ldots \ldots \ldots \ldots \ldots$ & 1.6013 \\
\hline
\end{tabular}

Note.-Table 4 is published in its entirety in the electronic edition of the Astrophysical Journal. A portion is shown here for guidance regarding its form and content.

with nightly extinction coefficients and transformed them to the Strömgren system with yearly mean transformation coefficients. Further information on the telescope, photometer, observing procedures, and data reduction techniques employed with the T11 APT can be found in Henry (1999) and Eaton et al. (2003).

A typical observation with the APT, termed a "group observation," consists of several measurements of the program star, each of which are bracketed by measurements of a comparison star or stars. The group observations typically take 515 minutes to complete, depending on the number of comparison stars in the group and the integration times for each star. The individual differential magnitudes determined within each group are then averaged to create group means, which are usually treated as single observations. On most nights, our observations of HD 149026 were obtained and reduced in this manner, resulting in a total of 153 group mean differential observations between 2005 April 25 and June 15 UT.

However, on the nights of 2005 May 14 UT (JD 2,453,504) and June 6 UT (JD 2,453,527), we monitored HD 149026 and its primary comparison star repeatedly for approximately $7.5 \mathrm{hr}$ around the time of conjunction predicted by the RV observations and obtained 300 individual differential measures on each night. Rather than averaging the resulting individual differential magnitudes within each group on these monitoring nights, we have retained the individual observations to increase the time resolution of our brightness measurements to about $90 \mathrm{~s}$ during the transit search. A few of the monitoring observations from each night were discarded as isolated outliers, resulting from such factors as telescope vibration, poor centering, or moments of poor seeing.

Additional simultaneous monitoring observations were acquired on the night of 2005 June 9 UT (JD 2,453,530) with the T8, T10, and T11 APTs; 330 differential observations were obtained with each telescope. The T8 and T10 APTs are functionally identical to the T11 APT (Henry 1999; Eaton et al. 2003). These June 9 observations from the three APTs were averaged together into a single data stream, and a couple of isolated outliers were discarded. To increase the precision of all of our total of 973 differential magnitudes, we averaged the Strömgren $b$ and $y$ magnitudes into a single $(b+y) / 2$ passband. The resulting $973(b+y) / 2$ differential magnitudes are listed in Table 4.

All but a few of our 153 group mean $(b+y) / 2$ differential magnitudes fall outside of the transit window predicted from the RV observations. The standard deviation of these out-of-transit observations is $0.0015 \mathrm{mag}$, indicating that both HD 149026 and its comparison star are constant to the limit of precision of the APTs. A least-squares sine fit of those observations phased to the RV period gives an upper limit to the semiamplitude of any 


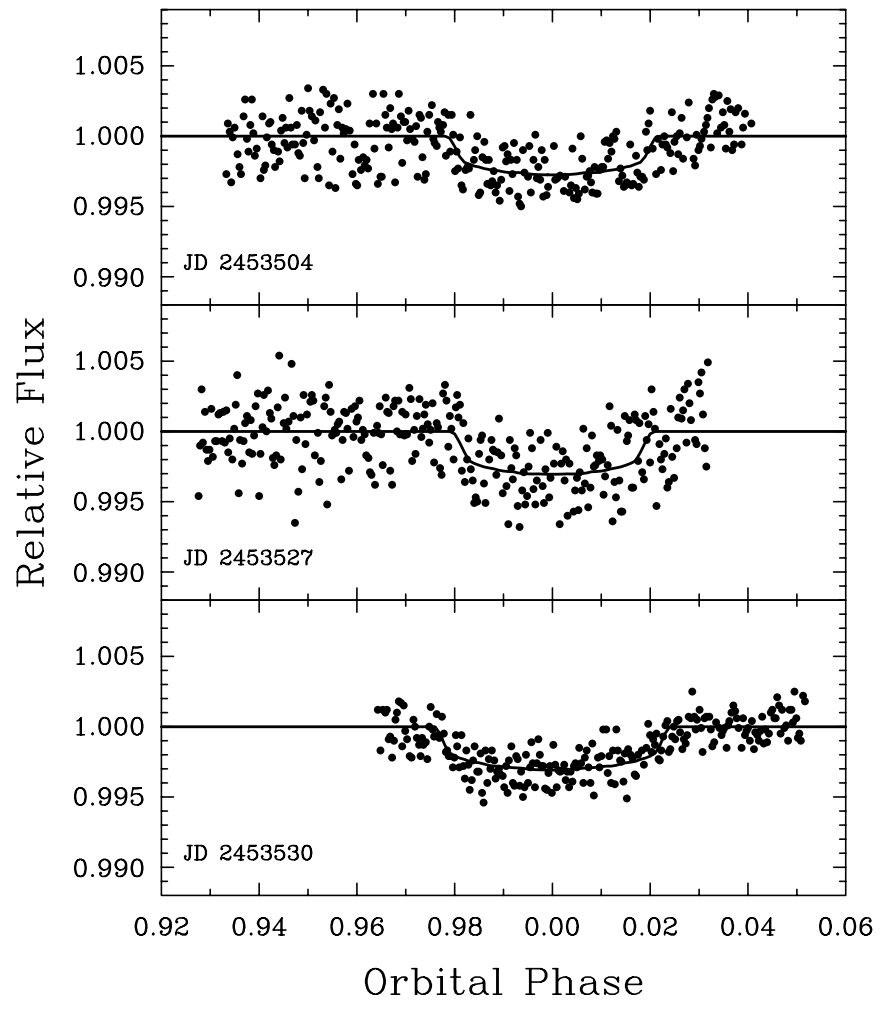

FIG. 3.-Photometric transits of HD 149026 in the Strömgren $(b+y) / 2$ passband observed with the T11 APT at Fairborn Observatory on 2005 May 14 UT (top) and 2005 June 6 UT (middle) and with the T8, T10, and T11 APTs on 2005 June 9 UT (bottom). The data from the three telescopes for the June 9 event have been averaged together to increase the precision. The solid curves represent the best-fit models given in Table 5, which result in a mean planetary radius of $0.725 R_{\mathrm{J}}$. This small planetary radius suggests that the planet has a substantial heavy-element core.

light variability on that period of only $0.0004 \pm 0.0002$ mag. Thus, starspots are unlikely to be the cause of the velocity periodicity in HD 149026. A few of the group mean observations, acquired just before dawn on the night of 2005 May $11 \mathrm{UT}$ (JD 2,453,501), fall within the early part of the transit window and suggest a slight dimming of around $0.003 \mathrm{mag}$. This was our first indication of possible transits in HD 149026. The geometric probability of transits in this system is approximately $15 \%$, computed from equation (1) of Seagroves et al. (2003).

Our three nights of monitoring observations around the predicted times of conjunction are plotted in Figure 3. The observations on all three nights confirm shallow transits in HD 149026. The Strömgren $(b+y) / 2$ differential magnitudes have been converted to linear intensity units relative to the mean outof-transit light level measured on each night. The monitoring observations have been phased with the 2.8766 day orbital period and the times of mid-transit determined for each event. The transits occurred approximately 0.05 days or $1.2 \mathrm{hr}$ earlier than

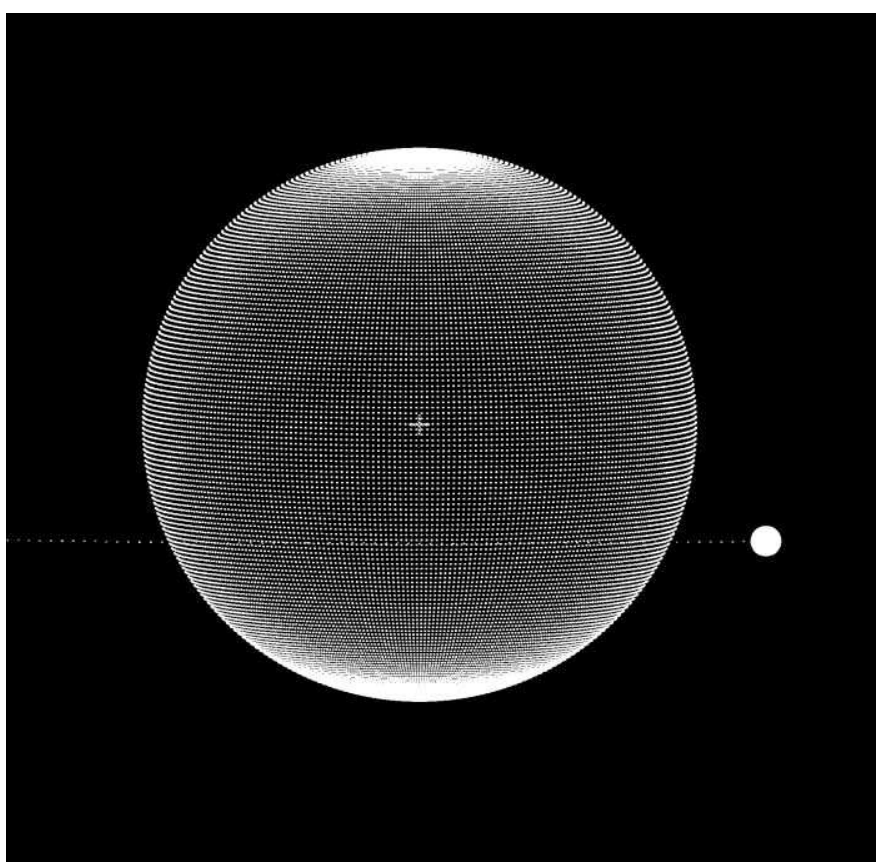

FIG. 4.-Depiction of the transit chord for the HD 149026 system based on the third transit solution. The ratio of the stellar to planetary radii is approximately $20: 1$, which when combined with the appropriate limb-darkening law results in the shallow observed transits of only 0.003 mag. It is unknown whether or not the planetary orbital plane is actually aligned with the stellar equatorial plane (see $\S 3)$.

predicted from the spectroscopic orbital solution, but within the limits of its uncertainty window. The observed duration of the transits is just over 0.04 phase units or approximately $3 \mathrm{hr}$, while the observed depth is only $0.003 \mathrm{mag}$, significantly less than the 0.007 mag events we were expecting from the stellar radius of $1.45 R_{\odot}$ (Table 1 ) and an anticipated planetary radius of $1.14 R_{\mathrm{J}}$, corresponding a gas giant planet with no core (see $\S 5$ ).

We modeled the three sets of transit observations separately with the eclipsing binary light curve synthesis software of Bradstreet \& Steelman (2002) to determine the planetary radius and orbital inclination, as well as the times of mid-transit. The stellar mass, radius, and effective temperature, along with the planetary mass, orbital period, and eccentricity, were fixed at the values given in Tables 1 and 3, respectively. A linear limbdarkening coefficient of 0.61 was used (van Hamme 1993), appropriate for the stellar $T_{\text {eff }}$ and $\log g$ values from Table 1 and the Strömgren $(b+y) / 2$ bandpass of the observations. No attempt was made to model the $b$ and $y$ light curves separately since no color change was detectable during the transit and also because the light curves in the individual bandpasses exhibited more scatter. Our best-fit time of mid-transit, planetary radius, and orbital inclination, as well as the rms of each solution, are

TABLE 5

Photometric Solutions For HD 149026b

\begin{tabular}{|c|c|c|c|c|c|c|}
\hline Transit & APT & $\begin{array}{l}\text { Date } \\
\text { (UT) }\end{array}$ & $\begin{array}{l}T_{\text {mid }} \\
(\mathrm{HJD})\end{array}$ & $\begin{array}{c}\text { Planetary Radius } \\
\left(R_{\mathrm{J}}\right)\end{array}$ & $\begin{array}{l}\text { Inclination } \\
\text { (deg) }\end{array}$ & $\begin{array}{l}\mathrm{rms} \\
(\mathrm{mag})\end{array}$ \\
\hline 1 & 11 & 2005 May 14 & $2,453,504.865$ & 0.705 & 84.6 & 0.0017 \\
\hline 2 & 11 & 2005 Jun 6 & $2,453,527.864$ & 0.759 & 84.1 & 0.0021 \\
\hline 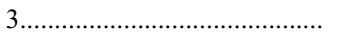 & $8,10,11$ & 2005 Jun 9 & $2,453,530.751$ & 0.719 & 86.2 & 0.0012 \\
\hline Weighted mean ..................... & $\ldots$ & .. & $\cdots$ & 0.725 & 85.3 & $\ldots$ \\
\hline
\end{tabular}




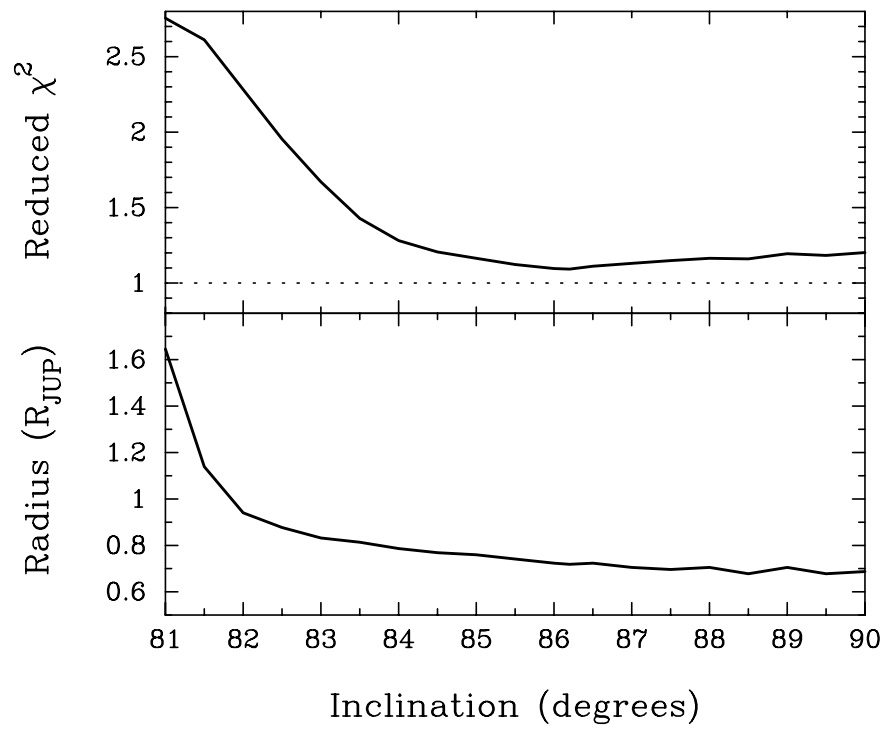

FIG. 5.- Reduced $\chi_{\nu}^{2}$ values (top) for the best-fit planetary radius over a range of assumed inclinations computed for the 2005 June 9 transit. As the inclination decreases, the best-fit planetary radius must increase (bottom) to compensate. These results demonstrate that the observations are incompatible with grazing or near-grazing transits of a larger planet.

listed in Table 5 . The weighted means of the planetary radius and the inclination are $(0.725 \pm 0.05) R_{\mathrm{J}}$ and $85^{\circ} .3 \pm 1.0$, respectively, where the third solution was given twice the weight of the others because of its significantly smaller rms scatter, and the radius is relative to Jupiter's equatorial radius, $R_{\mathrm{J}}=7.15 \times$ $10^{9} \mathrm{~cm}$. The error bars include the effects of the uncertainties in the assumed parameters, the most significant of which is the uncertainty in the stellar radius. Our best-fit models for each transit are shown by the solid curves in the three panels of Figure 3. The best-fit model of the third transit, which has the most precise observations, is shown pictorially in Figure 4 . The ratio of the stellar to planetary radius is approximately $20: 1$, resulting in the observed shallow transits of only 0.003 mag.

Because of the relatively large stellar radius and the short planetary orbital period, transits would occur for inclinations down to a limit of about $81^{\circ}$, where the center of the planet would just graze the stellar limb. However, grazing or neargrazing events result in much poorer fits to the photometric observations, as shown in the top panel of Figure 5, where we have plotted the reduced $\chi_{\nu}^{2}$ of the best-fit model of the third transit for a range of assumed inclinations from $81^{\circ}$ to $90^{\circ}$. As shown in the bottom panel of Figure 5, the best-fit planetary radius must increase as the inclination decreases, to compensate for crossing the star farther and farther from the center of the stellar disk. As the transits become partial below about $82^{\circ}$, the planetary radius must increase dramatically to maintain the observed transit depth. However, the transit duration for these grazing or near-grazing transits becomes much shorter than the observed duration of the transit, resulting in the much higher reduced $\chi_{\nu}^{2}$ values for the best fits seen in the top panel of Figure 5. Thus, the observations are clearly incompatible with grazing or near-grazing transits of a larger planet.

\section{COMPARISON WITH MODEL RADII}

Both the mass and the radius of HD 149026 are considerably smaller than those of the other known transiting extrasolar planets. Having estimated the planetary radius $R=(0.725 \pm$ $0.05) R_{\mathrm{J}}$ and mass $M=(0.36 \pm 0.04) M_{\mathrm{J}}$ by fitting the $\mathrm{RV}$ and transit photometry data, we are in a position to compare the results with theoretical evolutionary models. To do this, we use the results of Bodenheimer et al. (2003), who computed sequences of models for isolated planets ranging in mass from $0.11 M_{\mathrm{J}}$ to $3.0 M_{\mathrm{J}}$. Separate sequences were computed for models that contained and did not contain solid, constant-density ( $\rho=5.5 \mathrm{~g} \mathrm{~cm}^{-3}$ ) cores. Adopting the stellar and orbital parameters listed in Tables 1 and 3 and assuming a Bond albedo $A=$ 0.3 , we estimate an effective temperature for the planet of $T_{\text {eff }}=$ $1540 \mathrm{~K}$. This value is uncertain to $10 \%$ because the albedo is not known. The planet falls on the borderline between classes IV (typical albedo 0.03) and V (typical albedo 0.55) of Sudarsky et al. (2000). The corresponding uncertainty in the theoretical radius is roughly $5 \%$. Under these conditions, the models of Bodenheimer et al. (2003) predict a planetary radius of $R=$ $1.14 R_{\mathrm{J}}$ for an object of purely solar composition, and $R=0.97 R_{\mathrm{J}}$ for a planet with a $20 M_{\oplus}$ constant-density $\left(5.5 \mathrm{~g} \mathrm{~cm}^{-3}\right)$ core. Clearly, in order to explain the radius of HD 149026b, a substantial enrichment in heavy elements above solar composition is required. The mean density of the planet, $1.17 \mathrm{~g} \mathrm{~cm}^{-3}$, is 1.7 times that of Saturn, which itself has roughly $25 \%$ heavy elements by mass. On the other hand, the planet is not composed entirely of water ice or olivine, or else the radius would be $0.43 R_{\mathrm{J}}$ or $0.28 R_{\mathrm{J}}$, respectively (Guillot et al. 1996; Guillot 2005).

We have therefore computed new sequences of contracting and cooling planetary models of mass $0.36 M_{\mathrm{J}}$ with the code described in Bodenheimer et al. (2003), under the assumption that that planet is made up of a core of uniform density composed entirely of elements heavier than helium, and an envelope composed primarily of fluid with solar composition. It is of course possible that some of the heavy elements are not in the core but are dissolved in the envelope; however, the effects on the computed mean density will be similar (Guillot 2005). The calculations were started at a radius of 2-3 times the present value and were run up to an age of $2 \mathrm{Gyr}$, the estimated age of HD 149026. The planet is heated at the surface to $1500 \mathrm{~K}$ during the entire evolution. The surface boundary conditions are those for a gray radiative photosphere with a Rosseland mean opacity. The deduced radii are therefore approximate but nevertheless in reasonable agreement with more detailed calculations with nongray photospheres (Chabrier \& Baraffe 2000; Burrows et al. 2003, 2004; Chabrier et al. 2004). The envelope opacities in the outer radiative zone are purely molecular (R. Freedman 2003, private communication) and do not take into account the possible effects of clouds. The equation of state in the envelope is calculated from the tables of Saumon et al. (1995).

The results are surprising. Assuming a core density $\rho_{c}=$ $10.5 \mathrm{~g} \mathrm{~cm}^{-3}$, the estimated mean core density of Saturn (Marley 1999), we find (for $M=0.36 M_{\mathrm{J}}$ and $R=0.725 R_{\mathrm{J}}$ ) a solid core mass of $67 M_{\oplus}$. If $\rho_{c}=5.5 \mathrm{~g} \mathrm{~cm}^{-3}$, the required core mass rises to $78 M_{\oplus}$. For these two assumed core densities, which probably bracket the actual mean core density that would be obtained with a more detailed compressible equation of state for the core, the results for the derived core mass as a function of observed radius are given in Table 6 for a mass of $0.36 M_{\mathrm{J}}$. The interpolated core mass lies between entries A and B in Table 6 for $\rho_{c}=10.5 \mathrm{~g} \mathrm{~cm}^{-3}$, and between entries $\mathrm{C}$ and $\mathrm{D}$ for $\rho_{c}=5.5 \mathrm{~g} \mathrm{~cm}^{-3}$.

For both cases of assumed central density, the core comprises the majority of the planetary mass, indicating that despite its mass and orbital period, the planet is qualitatively more similar in structure to Neptune than it is to Saturn or Jupiter. Furthermore, the conclusion that the core is large seems secure. Even if the observed radius is increased by $2 \sigma$ to $0.825 R_{\mathrm{J}}$, the core 
TABLE 6

Model Radius and Core Mass

\begin{tabular}{|c|c|c|}
\hline \multicolumn{2}{|c|}{ Radius $\left(R_{\mathrm{J}}\right.$, EQUatoRial $)$} & \multirow{2}{*}{$\begin{array}{l}\text { Core Mass } \\
\qquad\left(M_{\oplus}\right)\end{array}$} \\
\hline$\rho_{c}=10.5 \mathrm{~g} \mathrm{~cm}^{-3}$ & $\rho_{c}=5.5 \mathrm{~g} \mathrm{~cm}^{-3}$ & \\
\hline 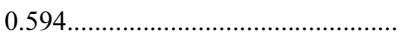 & $0.662(\mathrm{C})$ & 89.3 \\
\hline 0.681 (A) & 0.745 (D) & 74.5 \\
\hline 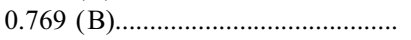 & 0.818 & 60.0 \\
\hline 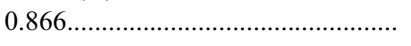 & 0.905 & 43.6 \\
\hline
\end{tabular}

Note.-A-B and $\mathrm{C}-\mathrm{D}$ bracket the interpolated core masses for the respective $\rho_{c}($ see $\S 5)$.

mass turns out to be $50.5 M_{\oplus}$ in the case of $\rho_{c}=10.5$ and $58.7 M_{\oplus}$ in the case of $\rho_{c}=5.5 \mathrm{~g} \mathrm{~cm}^{-3}$.

Note that the radii computed here do not include an effect pointed out by Burrows et al. (2003), namely, that the radius deduced from observations of a transit is larger by about $10 \%$ (for HD 209458b) than the theoretical "photospheric" radius because of the oblique viewing angle. For the lower mass planet HD 149026b, this effect could be even larger. If the planet radius is actually smaller than $0.7 R_{\mathrm{J}}$, then clearly the required enhancement of heavy elements would be even larger than quoted here.

\section{DISCUSSION}

The N2K consortium was established to survey "the next 2000 " closest and brightest high-metallicity stars. Here we describe a transiting Saturn-mass planet from the N2K consortium with an orbital period of 2.8766 days orbiting the $V=8.15$ star HD 149026. The planet was initially identified with Doppler observations from Subaru and confirmed with Keck observations. Remarkably, HD 149026 only dims by 0.003 mag during the $3 \mathrm{hr}$ transit of the planet. The photometric transit depth, together with the stellar radius of $1.45 R_{\odot}$, provides a planet radius of only $0.725 R_{\mathrm{J}}$. Four of seven Keck RVs were serendipitously obtained during transit. These four RVs exhibit the RM effect and provide an independent confirmation of the planetary transit.

With a radius of only $0.725 R_{\mathrm{J}}$, HD $149026 \mathrm{~b}$ seems anomalously small and emphasizes the diversity of extrasolar planet characteristics. Indeed, two of the three transits around bright stars (HD 209458, TrES-1, and HD 149026) are "anomalous." HD 209458 is too big, HD 149026 is too small; only TrES-1 conformed to prior expectations. These anomalies indicate a widerthan-expected diversity of planetary types and may also point to the need for models that include a wider variety of physical and atmospheric phenomena.

The small photometric depth of the transit underscores the most novel property of HD 149026b: its large core. The apparent presence of the large core has a number of potentially interesting ramifications for the theory of planet formation. First, it would be difficult to form this giant planet by the gravitational instability mechanism (Boss 2004), and by corollary, it likely illustrates a dramatic outcome of the core-accretion scenario (e.g., Hubickyj et al. 2004 and references therein). Among the giant planets in our solar system, Neptune is the hardest to account for within the framework of gravitational instability. In that picture, Neptune began as a $\sim 3 M_{\mathrm{J}}$ Jeans-unstable fragment. As the massive, cool proto-Neptune underwent Kelvin-Helmholtz contraction, its burden of solid grains settled to the central regions to form a core. As this was occurring, it is suggested (Boss 2003) that a nearby massive star photoevaporated the outer layers, leaving at the end of the day a $17 M_{\oplus}$ remnant of heavy elements, if a solar composition is assumed.

In order for this scenario to work for HD 149026b, the initial twice-solar metallicity fragment would need to have a total mass of order $6 M_{\mathrm{J}}$ in order to give rise to a $67 M_{\oplus}$ core, which is the total mass of solid components in the fragment. For such a large protoplanet, the settling time for the solid grains is considerably longer than the Kelvin-Helmholtz contraction time for the envelope. Furthermore, near-complete photoevaporation of a $6 M_{\mathrm{J}}$ protoplanet is difficult to accomplish.

However, the large core of HD 149026b also presents difficulties for conventional models of core accretion. In the coreaccretion theory, which was developed in the context of the minimum-mass solar nebula, it is difficult to prevent runaway gas accretion from occurring onto cores more massive than $30 M_{\oplus}$, even if abundant infalling planetesimals are heating the envelope and delaying the Kelvin-Helmholtz contraction that is required to let more gas into the planet's Hill sphere. The current structure of HD 149026b suggests that it was formed in a gas-starved environment, yet presumably enough gas was present in the protoplanetary disk to drive migration from its probable formation region beyond 1 or $2 \mathrm{AU}$ from the star inward to the current 2.87 day orbit.

We note, however, that the buildup of the large core mass was aided by the high-metallicity of the protoplanetary gas. In the standard theory, the isolation mass of a protostellar core scales as $M_{\text {iso }} \propto\left(a^{2} \sigma\right)^{3 / 2} M_{*}^{-1 / 2}$, where $\sigma$ is the surface density of solids in the disk and $a$ is the distance from the star, which has mass $M_{*}$. We assume that $\sigma$ scales as $M_{*} Z$. The final core mass, after further accretion of gas and solids, is about $\sqrt{2} M_{\text {iso }}$ (Pollack et al. 1996). For a twice-solar metallicity disk around a $1.3 M_{\odot}$ star, this implies a factor of 3.7 increase in $M_{\text {iso }}$ and $M_{\text {core }}$ as compared to their values in the minimum-mass solar nebula. But at least a factor of 3 increase in $\sigma$ above that in the minimummass solar nebula is required for the core accretion model to produce solar system giant planets in a time comparable to the lifetime of protoplanetary disks. As an example, we use the result of Hubickyj et al. (2004) for the formation of Jupiter at 5.2 AU around the Sun with $\sigma=10 \mathrm{~g} \mathrm{~cm}^{-2}$. They obtain $M_{\text {iso }}=$ $11.4 M_{\oplus}$ and $M_{\text {core }}=16.2 M_{\oplus}$. Scaling from that result, a plausible $M_{\text {core }}$ for HD $149026 \mathrm{~b}$ forming at $5.2 \mathrm{AU}$ is close to $60 M_{\oplus}$. Even if no further accretion of solids occurs after the isolation mass is reached, $M_{\text {core }}$ would be $42 M_{\oplus}$. Planet formation models with shorter accretion timescales may be key to understanding the formation of HD 149026b (see Alibert et al. 2005).

Several scenarios for forming a Saturn-mass object with a $\sim 67 M_{\oplus}$ core can be envisioned. The planet may have migrated inward when it was less massive than at present and become stranded at the 2:1 resonance with the so-called X-point of the protostellar disk (e.g., Shu et al. 1994; see also the suggestion in Lin et al. 1996). After the planetary core was stranded, gas migrating through the disk would climb the magnetic field lines onto the star, whereas planetesimals would cross through the $\mathrm{X}$-point and be pushed further inward by the disk torque. Some of these planetesimals would accrete onto the stranded planet, increasing its compositional fraction of heavy elements (Ward 1997).

Alternately, a planet with a large core could be formed through a giant impact scenario between two isolation-mass embryos. Such an object could then either migrate inward via conventional type II migration or perhaps have been scattered into a highly eccentric orbit by interactions with other planets in the system. In the case of a scattering history, the orbital plane of the planet was likely to have been initially misaligned with 
the spin axis of the star. This scenario may soon find support as more in-transit RVs that exhibit the RM effect on the stellar lines are obtained (e.g., Winn et al. 2005).

There were two necessary components for this transit detection. First, the initial Doppler reconnaissance at Subaru and Keck detected the low-mass, short-period planet and provided accurate transit predictions. Second, the robotic telescopes at Fairborn Observatory were available to obtain baseline photometry and to carry out millimagnitude-precision photometric observations at the predicted transit times. This approach, combining a quick-look Doppler survey with targeted high-precision photometry, has turned out to be an efficient way to detect transiting planets around bright stars; both HD 209458 and HD 149026 were discovered with this strategy.

The small radius of this transiting planet bears upon the expected detection rate by wide-field photometric transit surveys of bright stars. Such a small transit depth would likely be missed with the typical photometric precision of $0.01 \mathrm{mag}$. Another factor that may negatively impact detection rates for widefield transit surveys is the Malmquist bias. Because of this effect, subgiants are preferentially included in magnitude-limited samples. The larger stellar radii of these stars reduces the transit depth and demands millimagnitude observational precision. Of the four stars with short-period planets detected in the N2K program, all have somewhat extended radii. HD 88133 has a radius of $1.93 R_{\odot}$ and a planet mass of $M \sin i=0.25 M_{\mathrm{J}}, \mathrm{HD}$ 149143 has a radius of $1.49 R_{\odot}$ and a planet mass of $1.32 M_{\mathrm{J}}$, HD 109749 has a stellar radius of $1.24 R_{\odot}$ and a planet mass of $0.27 M_{\mathrm{J}}$, and HD 149026 has a radius of $1.45 R_{\odot}$ and a planet mass of $0.36 M_{\mathrm{J}}$. While the transit probabilities for all four of these detections exceed the canonical $10 \%$ transit probability because of the distended stellar radii, the predicted transit depths are only a fraction of the transit depth observed for HD 209458.

At one time, photometric surveys were predicted to detect hundreds of transiting planets per month (Horne 2003). Instead, transit detection has turned out to be harder than anticipated, perhaps because the high-precision regime is technically difficult for any technique and because the diversity of planet characteristics continues to surprise us. The combination of the Doppler technique, which is sensitive to the gravitational effect of the planet, and targeted high-precision photometry provide complementary information that dramatically increases our understanding of extrasolar planets.

We thank Akito Tajitsu for his expertise and support of the Subaru HDS observations. We gratefully acknowledge the dedication and support of the Keck Observatory staff, in particular Grant Hill for support with HIRES. We thank the NOAO and NASA telescope assignment committees for generous allocations of telescope time. Data presented herein were obtained at the W. M. Keck Observatory from telescope time allocated to NASA through the agency's scientific partnership with the California Institute of Technology and the University of California. The Observatory was made possible by the generous financial support of the W. M. Keck Foundation. We thank the Michaelson Science Center for travel support through the KDPA program. D. A. F. is a Cottrell Science Scholar of the Research Corporation. We acknowledge support from NASA grant NNG05G164G (to D. A. F.), NASA grant NCC5-511 and NSF grant HRD 97-06268 (to G. W. H.), NASA grant NAG575005 (to G. W. M.), NSF grant AST 99-88358 and NASA grant NAG5-4445 (to S. S. V.), NASA grant NAG5-13285 (to P. B.), and NASA grant NNA04CC99A (to G. L.). G. W. H. also acknowledges timely support by David Bradstreet for requested modifications to his light-curve analysis software to fit the unexpectedly shallow transits in HD 149026 and thanks Stephen Henry for assistance in the preparation of Figure 5. D. M. is supported by FONDAP 15010003. This research has made use of the SIMBAD database, operated at CDS, Strasbourg, France. The authors extend thanks to those of native Hawaiian ancestry on whose sacred mountain of Mauna Kea we are privileged to be guests. Without their generous hospitality, the Subaru and Keck observations presented herein would not have been possible.

\section{REFERENCES}

Alibert, Y., Mordasini, C., Benz, W., \& Winisdoerffer, C. 2005, A\&A, 434, 343 Alonso, R., et al. 2004, ApJ, 613, L153

Ammons, M., et al. 2005, ApJ, submitted

Baraffe, I., Chabrier, G., Barman, T. S., Allard, F., \& Hauschild, P. H. 2003, A\&A, 402, 701

Bodenheimer, P., Laughlin, G., \& Lin, D. N. C. 2003, ApJ, 592, 555

Bodenheimer, P., Lin, D. N. C., \& Mardling, R. 2001, ApJ, 548, 466

Boss, A. P. 2003, ApJ, 599, 577 2004, ApJ, 610, 456

Bradstreet, D. H., \& Steelman, D. P. 2002, BAAS, 34, 1224

Brown, T. M., Charbonneau, D., Gilliland, R. L., Noyes, R. W., \& Burrows, A. 2001, ApJ, 552, 699

Burrows, A., Hubeny, I., Hubbard, W. B., Sudarsky, D., \& Fortney, J. J. 2004, ApJ, 610, L53

Burrows, A., Sudarsky, D., \& Hubbard, W. B. 2003, ApJ, 594, 545

Butler, R. P., Marcy, G. W., Williams, E., McCarthy, C., Dosanjh, P., \& Vogt, S. S. 1996, PASP, 108, 500

Butler, R. P., Vogt, S. S., Marcy, G. W., Fischer, D. A., Wright, J. T., Henry, G. W., Laughlin, G., \& Lissauer, J. 2004, ApJ, 617, 580

Chabrier, G., \& Baraffe, I. 2000, ARA\&A, 38, 337

Chabrier, G., Barman, T., Baraffe, I., Allard, F., \& Hauschildt, P. 2004, ApJ, 603, L53

Charbonneau, D., Brown, T. M., Latham, D. W., \& Mayor, M. 2000, ApJ, 529, L45

DeMarque, P., Woo, J. H., Kim, Y. C., \& Yi, S. K. 2004, ApJS, 155, 667

Deming, D., Brown, T. M., Charbonneau, D., Harrington, J., \& Richardson, L. J. 2005, ApJ, 622, 1149

Eaton, J. A., Henry, G. W., \& Fekel, F. C. 2003, in The Future of Small Telescopes in the New Millennium, Vol. 2, The Telescopes We Use, ed. T. D. Oswalt (Dordrecht: Kluwer), 189

Fischer, D. A., \& Valenti, J. A. 2005, ApJ, 622, 1102

Fischer, D. A., et al. 2005a, ApJ, 620, 481

- 2005b, ApJ, submitted

Fuhrmann, K., Pfeiffer, M. J., \& Bernkopf, J. 1997, A\&A, 326, 108 1998, A\&A, 336, 942

Girardi, L., Bressan, A., Bertelli, G., \& Chiosi, C. 2000, A\&AS, 141, 371

Gonzalez, G. 1997, MNRAS, 285, 403

1998, A\&A, 334, 221 1999, MNRAS, 308, 447

Gonzalez, G., Laws, C., Tyagi, S., \& Reddy, B. E. 2001, AJ, 121, 432

Guillot, T. 2005, Annu. Rev. Earth Planet. Sci., 33, 493

Guillot, T., Burrows, A., Hubbard, W. B., Lunine, J. I., \& Saumon, D. 1996, ApJ, 459, L35

Guillot, T., \& Showman, A. P. 2002, A\&A, 385, 156

Henry, G. W. 1999, PASP, 111, 845

Henry, G. W., Baliunas, S. L., Donahue, R. A., Fekel, F. C., \& Soon, W. 2000a, ApJ, 531, 415

Henry, G. W., Marcy, G. W., Butler, R. P., \& Vogt, S. S. 2000b, ApJ, 529, L41

Horne, K. 2003, in ASP Conf. Ser. 294, Scientific Frontiers in Research on Extrasolar Planets, ed. D. Deming \& S. Seager (San Francisco: ASP), 361

Hubickyj, O., Bodenheimer, P., \& Lissauer, J. J. 2004, Rev. Mex. AA Ser. Conf., 22, 83

Ida, S., \& Lin, D. N. C. 2004, ApJ, 616, 567

Kambe, E., et al. 2002, PASJ, 54, 865

Laughlin, G., Marcy, G. W., Vogt, S. S., Fischer, D. A., \& Butler, R. P. 2005 , ApJ, 629, L121

Lin, D. N. C., Bodenheimer, P., \& Richardson, D. 1996, Nature, 380, 606

Marcy, G. W., Butler, R. P., Fischer, D. A., \& Vogt, S. S. 2004, in ASP Conf. Ser. 321, Extrasolar Planets: Today and Tomorrow, ed. J.-P. Beaulieu, A. Lecavelier des Etangs, \& C. Terquem (San Francisco: ASP), 3 
Marley, M. 1999, PASP, 111, 1591

Mayor, M., \& Queloz, D. 1995, Nature, 378, 355

Mayor, M., Udry, S., Naef, D., Pepe, F., Queloz, D., Santos, N. C., \& Burnet, M. 2004, A\&A, 415, 391

McArthur, B. E., et al. 2004, ApJ, 614, L81

Noguchi, K., et al. 2002, PASJ, 54, 855

Paulson, D. B., Saar, S. H., Cochran, W. D., \& Henry, G. W. 2004, AJ, 127, 1644

Perryman, M. A. C. 1997, The Hipparcos and Tycho Catalogues (ESA SP-1200; Noordwijk: ESA)

Pollack, J. B., Hubickyj, O., Bodenheimer, P., Lissauer, J. J., Podolak, M., \& Greenzweig, Y. 1996, Icarus, 124, 62

Queloz, D., et al. 2001, A\&A, 379, 279

Reid, I. N. 2002, PASP, 114, 306

Rivera, E., Lissauer, J., Butler, R. P., Marcy, G. W., Vogt, S. S., Fischer, D. A., Brown, T., \& Laughlin, G. 2005, ApJ, submitted

Robinson, S. E., et al. 2005, ApJ, submitted

Santos, N. C., Israelian, G., \& Mayor, M. 2001, A\&A, 373, 1019 2004a, A\&A, 415, 1153

Santos, N. C., Israelian, G., Mayor, M., Rebolo, R., \& Udry, S. 2003, A\&A, 398,363
Santos, N. C., et al. 2004b, A\&A, 426, L19

Sato, B., Kambe, E., Takeda, Y., Izumiura, H., \& Ando, H. 2002, PASJ, 54, 873

Saumon, D., Chabrier, G., \& van Horn, H. 1995, ApJS, 99, 713

Seagroves, S., Harker, J., Laughlin, G., Lacy, J., \& Castellano, T. 2003, PASP, 115,1355

Shao, M. 2004, Proc. SPIE, 5491, 328

Shu, F., Najita, J., Ostriker, E., Wilkin, F., Ruden, S., \& Lizano, S. 1994, ApJ, 429, 797

Sudarsky, D., Burrows, A., \& Pinto, P. 2000, ApJ, 538, 885

Valenti, J. A., \& Fischer, D. A. 2005, ApJS, 159, 141

van Hamme, W. 1993, AJ, 106, 2096

Vogt, S. S., Butler, R. P., Marcy, G. W., Fischer, D. A., Henry, G. W., Laughlin, G., Wright, J. T., \& Johnson, J. 2005, ApJ, in press

Wallace, L., Hinkle, K., \& Livingston, W. C. 1993, An Atlas of the Photospheric Spectrum from 8900 to $13,600 \mathrm{~cm}^{-1}$ (NSO Tech. Rep. 93-001; Tucson: NSO) Ward, W. 1997, ApJ, 482, L211

Winn, J. N., et al. 2005, ApJ, 631, 1215

Wittenmyer, R. A., et al. 2005, ApJ, 632, 1157 